\title{
Progress on the Relativistic Klystron Two-Beam Accelerator Prototype
}

\author{
G. A. Westenskow, D. E. Anderson, S. Eylon, E. Henestroza, \\ T. L. Houck, S. M. Lidia, D. L. Vanecek, and S. S. Yu
}

This paper was prepared for submittal to the 8th Workshop on Advanced Accelerator Concepts

Baltimore, Maryland

July 5-11, 1998

July 5, 1998

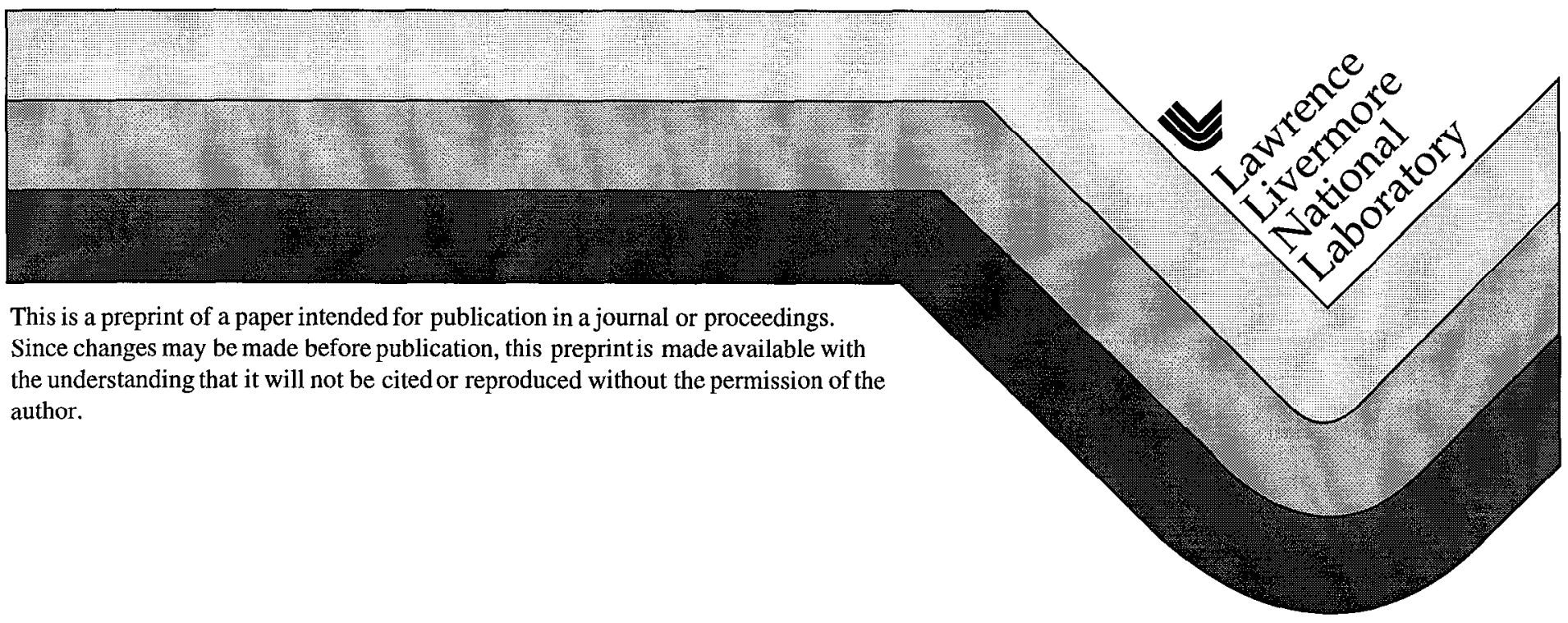




\section{DISCLAIMER}

This document was prepared as an account of work sponsored by an agency of the United States Government. Neither the United States Government nor the University of California nor any of their employees, makes any warranty, express or implied, or assumes any legal liability or responsibility for the accuracy, completeness, or usefulness of any information, apparatus, product, or process disclosed, or represents that its use would not infringe privately owned rights. Reference herein to any specific commercial product, process, or service by trade name, trademark, manufacturer, or otherwise, does not necessarily constitute or imply its endorsement, recommendation, or favoring by the United States Government or the University of California. The views and opinions of authors expressed herein do not necessarily state or reflect those of the United States Government or the University of California, and shall not be used for advertising or product endorsement purposes. 


\title{
Progress on the Relativistic Klystron Two-Beam Accelerator Prototype
}

\author{
G. A. Westenskow*, D. E. Anderson ${ }^{\dagger}$, S. Eylon ${ }^{\dagger}$, E. Henestroza ${ }^{\dagger}$, \\ T. L. Houck*, S. M. Lidia ${ }^{\dagger}$, D. L. Vanecek ${ }^{\dagger}$, and S. S. Yu ${ }^{\dagger}$ \\ *Lawrence Livermore National Laboratory, Livermore, CA 94551 \\ ${ }^{\dagger}$ Lawrence Berkeley National Laboratory, Berkeley, CA 94720
}

\begin{abstract}
The technical challenge for making two-beam accelerators into realizable power sources lies in the creation of the drive beam and in its propagation over long distances through multiple extraction sections. This year we have been constructing a $1.2-\mathrm{kA}, 1-\mathrm{MeV}$, electron induction prototype injector as a collaborative effort between LBL and LLNL. The electron source will be a 3.5"-diameter, thermionic, flat-surface cathode with a maximum shroud field stress of approximately $165 \mathrm{kV} / \mathrm{cm}$. Additional design parameters for the injector include a pulse length of over 120-ns flat top (1\% energy variation), and a normalized edge emittance of less than $200 \pi$-mm-mr. Planned diagnostics include an isolated cathode with resistive divider for direct measurement of current emission, resistive-wall and magnetic probe current monitors for measuring beam current and centroid position, capacitive probes for measuring $\mathrm{A}-\mathrm{K}$ gap voltage, an energy spectrometer, and a pepper-pot emittance diagnostic. Details of the injector, beam line, and diagnostics are presented.
\end{abstract}

\section{INTRODUCTION}

Induction accelerators are a unique source for high-current, high-brightness, electron beams. A collaboration between the Lawrence Livermore National Laboratory (LLNL) and Lawrence Berkeley National Laboratory (LBNL) has been studying if power sources based on the Relativistic Klystron Two-Beam Accelerator (RK-TBA) concept for several years $(1,2)$. A major technical challenge to the successful operation of a full scale RK-TBA is the transport of the electron beam through several hundred meters of narrow aperture microwave extraction structures and induction accelerator cells. Demanding beam parameters are required of the electron source, an induction injector, to achieve the transport goals. A test facility, called the RTA, has been established at LBNL (3) to verify the analysis used in the design study. The primary effort of the facility is the construction of a prototype RK-TBA subunit that will permit the study of technical issues, system efficiencies, and costing. In this paper, we will discuss the development of the RTA electron source and it's pulsed power system, which has recently been constructed and is now undergoing testing. 


\section{RTA GUN}

A major part of our effort during the past year has been towards the design of a low emittance electron source for RTA accelerator. We expect to produce an electron source with a much lower emittance than typical induction guns. The electron source will be a 3.5"-diameter, thermionic, flat-surface W-type cathode with a maximum shroud field stress of approximately $165 \mathrm{kV} / \mathrm{cm}$. An emission density of $20 \mathrm{~A} / \mathrm{cm}^{2}$ is required from the cathode to produce $1.2 \mathrm{kA}$ beam. The RTA gun, depicted in Figure 1, has 72 induction cores, each driven at $14 \mathrm{kV}$. The voltage across the A-K gap is $1 \mathrm{MV}$. The cores are segmented radially to reduce the individual aspect $(\Delta \mathrm{r} / \Delta \mathrm{z})$ ratio. The lower aspect ratio reduces the variation in core impedance during the voltage pulse simplifying the pulse forming network (PFN) design. Figure 2 is a photograph of the gun undergoing initial pulsed power tests.

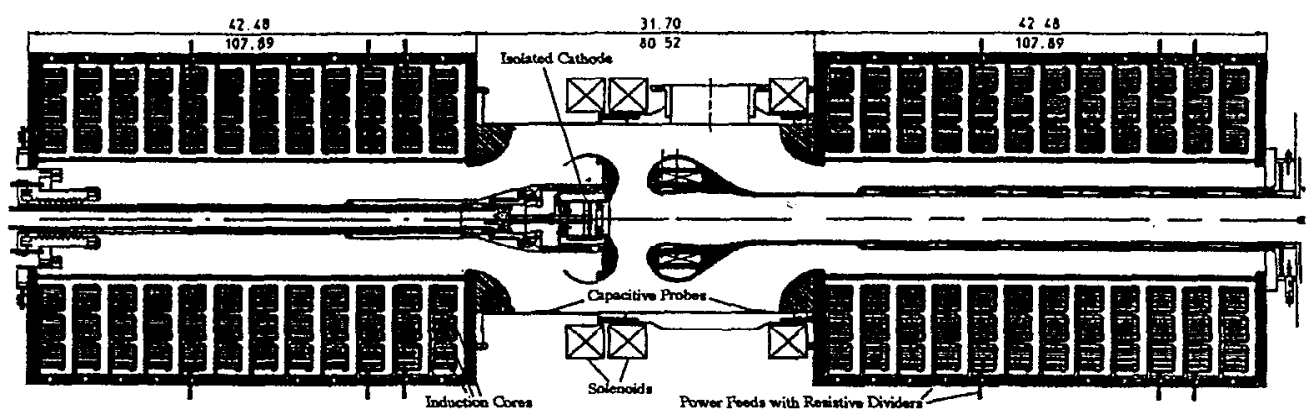

Figure 1. Depiction of the RTA electron gun.

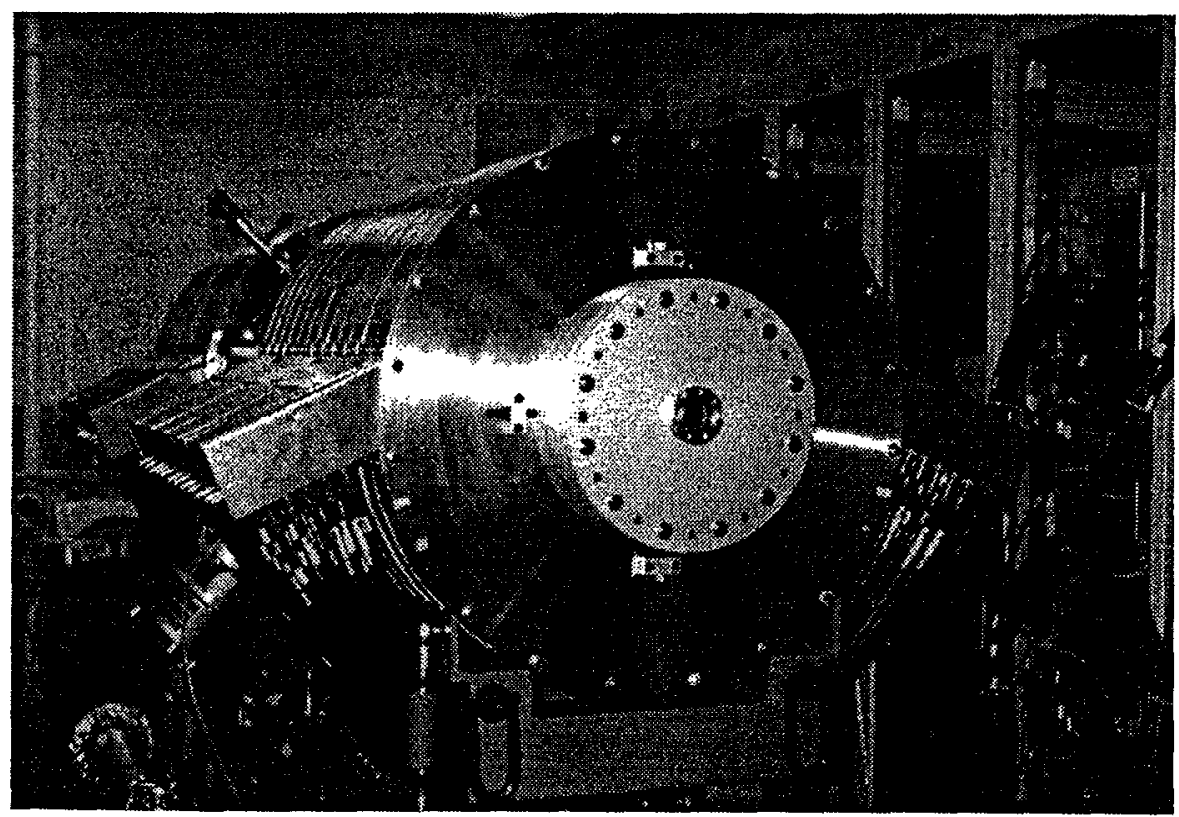

Figure 2. RTA 1-MV, 1-kA electron gun with pulse power system. 
We have done high-voltage tests on the gun. In operation a $500 \mathrm{kV}$ potential is developed across the two 30-cm-ID PYREX (4) insulators producing a $5.1 \mathrm{kV} / \mathrm{cm}$ average gradient along the insulator. The maximum fields at the triple points, the intersection of insulator, vacuum, and metal, is less than $3.5 \mathrm{kV} / \mathrm{cm}$. Maximum surface field in the cathode half of the gun electrode is about $85 \mathrm{kV} / \mathrm{cm}$. The maximum field is about $116 \mathrm{kV} / \mathrm{cm}$ on the anode stalk.

Initial beam focusing in the gun is accomplished by three large-bore air-core solenoids installed on the central pumping spool. The first solenoid is operated to null the magnetic field from the other solenoids at the cathode front surface. There are seven smaller solenoids located within the anode stalk to provide additional focusing to transport the beam to the end of the gun.

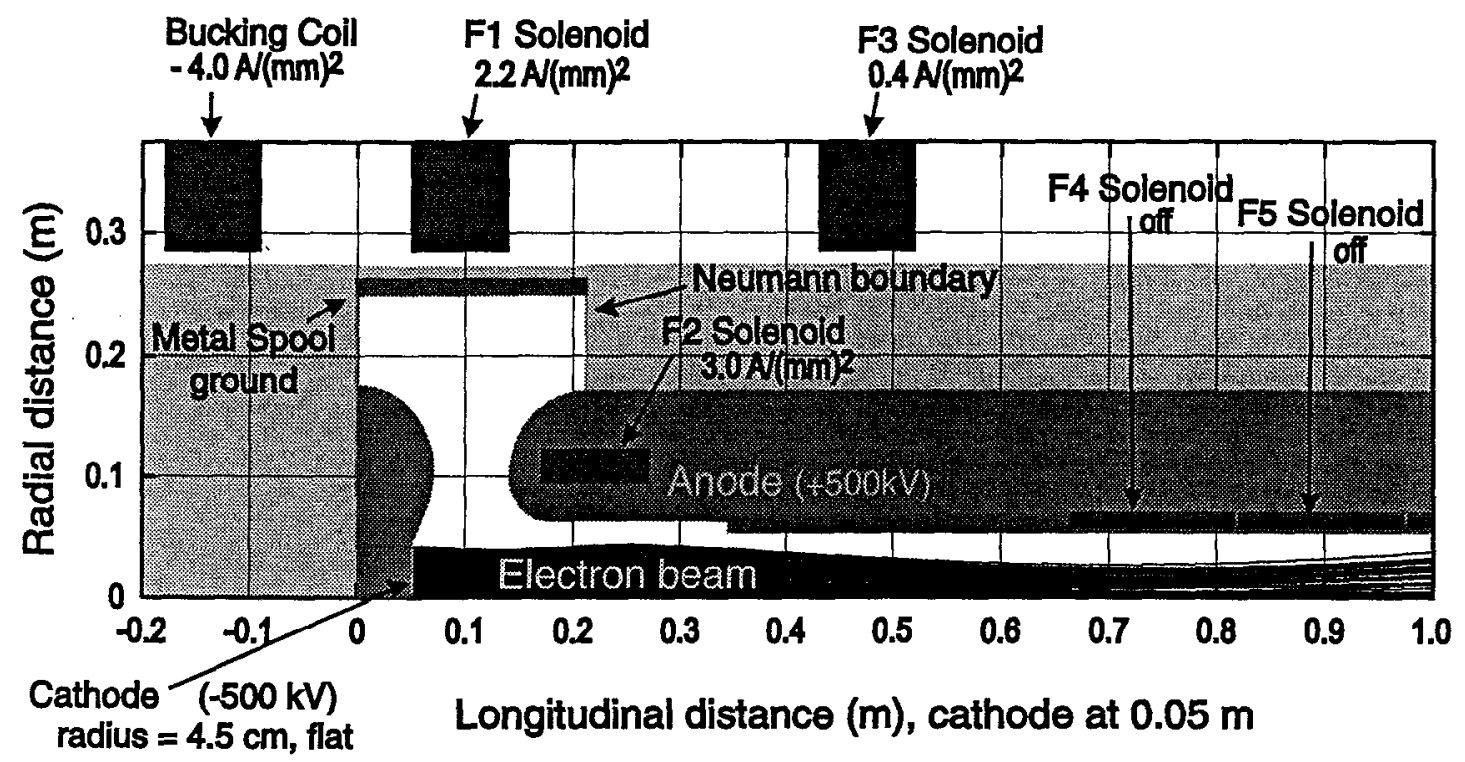

Figure 3. EGUN simulation of beam transport near the cathode of the RTA Gun.

\section{PULSED POWER SYSTEM FOR THE RTA GUN}

The pulsed power system for the gun consists of a $20-\mathrm{kV}$ High-Voltage Power Supply, 6-kJ Energy Storage Bank, two Command Resonant Charging (CRC) Chassis, 24 Switched Pulse Forming Networks, and four Induction Core Reset Pulsers, half of which is shown in Figure 4. Each PFN will drive a single 3-core induction cell of the gun. A sample pulse for a single cell with a $40 \Omega$ resistor simulating beam loading is shown in Figure 5.

Segmenting the core in the induction cell and driving the individual core segments avoids a high-voltage step-up transformer. This reduces the developmental effort needed to achieve a "good" flattop pulse (minimal energy variation) with fast risetime and improves the efficiency of the overall pulsed power system. Our system of lowvoltage PFNs driving multiple core induction cells is similar to the system envisioned for the extraction section in the full scale RK-TBA design. For the gun core material, 


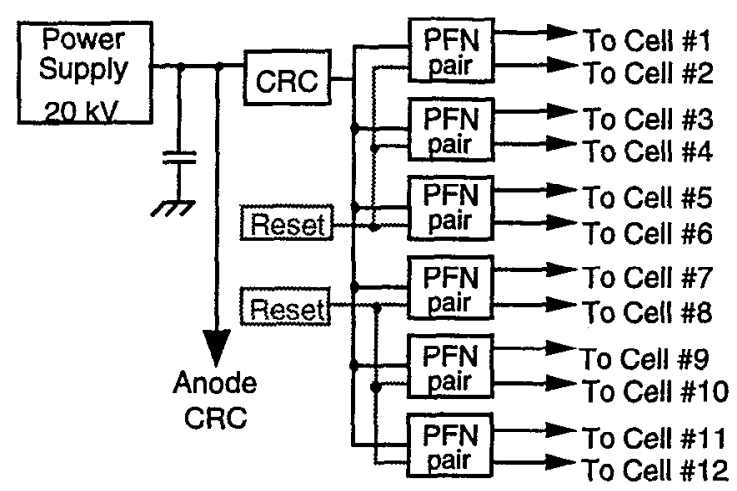

Figure 4. Block diagram of the pulsed power system for the cathode half of the RTA gun.

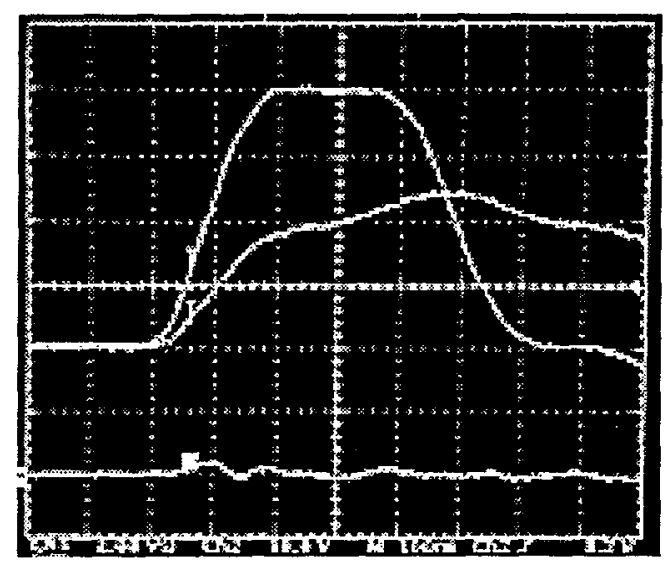

Figure 5. Oscilloscope trace of pulsed gap voltage applied to a single induction cell. Top trace is voltage $(10 \mathrm{kV} / \mathrm{div})$ and middle trace is current $(4 \mathrm{kA} / \mathrm{div})$. Time scale : $100 \mathrm{~ns} / \mathrm{div}$.

we choose $20-\mu$ m-thick 2605 SC METGLAS. For the RTA extraction section we will use a lower loss 2714AS METGLAS for the induction cores.

Design of the switched PFNs follows easily from published METGLAS core loss data (5). For the RTA induction cells, a flux swing of $2.6 \mathrm{~T}$ in $400 \mathrm{~ns}$ (FWHM) results in a magnetization rate of $6.5 \mathrm{~T} / \mu \mathrm{s}$. At this rate, a loss density of $1800 \mathrm{~J} / \mathrm{m}^{3}$ translates into $30 \mathrm{~J}$ lost in a cell with $16.7 \times 10^{3} \mathrm{~cm}^{3}$ of $2605 \mathrm{SC}$ METGLAS. For a cell input voltage of $14 \mathrm{kV}$ applied for $400 \mathrm{~ns}$, these losses require that $5900 \mathrm{~A}$ be supplied to the three radial cores. An additional $3600 \mathrm{~A}$ is required to supply beam current (1200 A x 3 cores/cell), resulting is a total current of $9 \mathrm{kA}$. The required drive impedance for a cell is then $1.5 \Omega$, which is provided by the PFN module shown in Figure 4.

An area of concern was the variation of the energy loss for different METGLAS cores. Experience (6) with the 72 cores in the gun leads us to believe that for a large relativistic klystron, matching cell cores should permit acceptable energy loss variation. 


\section{GUN VOLTAGE WAVEFORM}

Achieving the fast risetime necessary to minimize the volt-seconds required for the injector cores presented a challenge (see Figure 5). Budget constraints coupled with the large availability of EEV CX1538 thyratrons from the ATA program at LLNL made these tubes an attractive option. However, their poor time rate of current change ( $4 \mathrm{kA} / \mu \mathrm{s}$ rating) made them questionable for this application, which requires about 40 $\mathrm{kA} / \mu \mathrm{s}$. A variety of techniques were tried to decrease the risetime. In a $1.5 \Omega$ system, stray circuit inductances must be maintained at or below $100 \mathrm{nH}$ to achieve a $10-90 \%$ risetime of 150 ns. This was accomplished by placing the thyratron between two current sheets connecting the PFN output to the output cables. The ionization time of the thyratron was substantially reduced by applying a 1-2 A pre-pulse to the keep-alive grid 300-400 ns prior to the arrival of the main control-grid pulse. Faster risetimes were achieved with Triton F-130 ceramic thyratrons. An upgrade of the current thyratrons in the gun pulsed power system should allow us to achieve the design 100-ns risetime.

At the $1-\mathrm{MV}, 1.2-\mathrm{kA}$ operating conditions we hope to produce a $\pm 1 \%$ gun voltage flat waveform for $120 \mathrm{~ns}$. We will need to adjust the number of turns in appropriate sections of individual PFNs to achieve this goal. Insertion of ferrite material in the center of the inductors coils will allow additional small corrections to the waveform. There are 24 EEV CX1538 thyratrons used in the pulsed power system for the gun. Since the turn-on time is voltage dependent for each of the tubes, we are adjusting the trigger time of each individual tube.

\section{DIAGNOSTICS FOR THE RTA GUN}

A variety of diagnostics will be used to determine the performance of the gun, both permanently installed monitors for general operations and temporary diagnostics specific to the injector commissioning and troubleshooting. The majority of the diagnostics will be installed after the gun. The first $1.4 \mathrm{~m}$ of beam line after the gun will include two beam position and current monitors to allow the offset and angle of the beam at the exit of the injector to be measured. A pop-in probe will be incorporated in a pumping port to allow the beam profile to be viewed. We plan to use a pepperpot emittance diagnostic to help characterize the beam.

\section{Current Measurements}

An accurate measurement of the emitted current from the cathode is required both for determining the performance of the gun and benchmarking codes. We have electrically isolated the cathode from the stalk, forcing the current to flow through several parallel, 0.25 inch wide, strips of $25-\mu \mathrm{m}$ thick nichrome foil that act as currentviewing resistors. The potential drop across the foil is measured and the current inferred. To improve the time response a parallel-strip shunt geometry is used where 
the foil is folded on itself to increase the resistance while lowering the series inductance.

Two different methods (7) will be used to determine the beam current and centroid position in the region between the gun and the electron dump. Magnetic pickup (B-dot). loops will determine the time derivative of the current pulse. The voltage induced on the loop can then be integrated to recover the current. The second method uses resistive wall current monitors to measure the potential drop of the return wall current across a known resistance generating a signal proportional to the current. Our monitors will consist of $25-\mu \mathrm{m}$ thick nichrome foil that spans a short insulated break around the circumference of the beam tube wall and have a total resistance of a few milliohms. The slow decay in the output signal for this diagnostic can be corrected through compensation circuits, either passive or active. The resistive wall current monitors have the advantage of producing a signal proportional to the current. However, the frequency response is less than that of B-dot loops.

The beam dump will be electrically isolated through nichrome film (current-viewing resistors) similar to the cathode to allow for the measurement of the total current deposited in the dump..

The errors inherent in the above monitors for off-axis beams are easily calculated and not expected to be an issue. The major concern is in the calibration of the diagnostics. The nominal tolerance for commercial components used in the monitors would limit accuracy to about 5\%. By calibrating complete systems, i.e. the individual diagnostic with cables, integrators, and compensation circuits, as applicable, wc hope to achieve accuracies approaching $1 \%$.

\section{Current Density Profile}

The current density profile will be measured using Cherenkov and/or optical transition radiation from intercepting foils. A primary concern with using foils is possible damage from beam energy deposition. Average heating of the foil can be controlled by adjusting the repetition rate of the injector. The difficulty is the single shot heating where material can be melted and ejected before the heat is conducted away. If the foils are sufficiently thin that the radiation generated by bremsstrahlung escapes, and assuming the beam pulses are short compared to the thermal conduction time, the temperature rise in the foil is approximately:

$$
\Delta \mathrm{T}=\frac{\mathrm{J} \Delta \mathrm{t}}{\rho \mathrm{C}_{\mathrm{p}} \mathrm{e}}\left[\frac{\mathrm{dT} \mathrm{e}}{\mathrm{dz}}\right]_{\mathrm{c}}
$$

where $J$ is the current density, $\Delta t$ is the pulse length, $\rho$ and $C_{p}$ are the density and specific heat of the foil, respectively, and $\left[\mathrm{dT}_{\mathrm{e}} / \mathrm{dz}\right]_{\mathrm{c}}$ is the collisional stopping power for the electrons. For a given foil material, the minimum beam radius at intercept can be determined from equation (2). The collisional stopping power is reasonably constant with energy for relativistic electrons. To avoid damage for a thin quartz foil, the beam diameter must be larger than $2 \mathrm{~cm}$ for a $1-\mathrm{kA}, 300-\mathrm{ns}$, relativistic electron beam. We anticipate using a number of foil materials, including kapton, quartz, graphite, tantalum, and tungsten. 
The light generated at the beam/foil interaction will be recorded using both gated and streak cameras. The streak camera will be used principally to determine if the properties of the foil and/or beam change during the pulse. The significant levels of energy deposited in the foil could affect the dielectric constant or generate a surface plasma that could be confused as a variation in beam parameters.

\section{A-K Voltage and Beam Energy}

Three different methods will be used to determine the A-K voltage and beam energy. The first method involves measuring the applied voltage to the induction cores at the connection of the power feeds to the induction cells. Capacitive $\mathrm{dV} / \mathrm{dt}$ pickup probes (7) are used for a more direct measurement of the A-K gap voltage and also to provide greater bandwidth with respect to the resistive dividers. We also hope to employ a conventional energy spectrometer comprised of an on-axis collimator, dipole magnet, scintillator, and viewing port to directly measure the beam energy.

\section{Emittance Measurement}

Measuring the beam emittance is expected to be very difficult as the beam is highly space charged dominated. A pepperpot emittance diagnostic is being constructed. The effect of space charge can be appreciated by considering the envelope equation for a round, uniform beam in a drift region:

$$
\frac{d^{2} R}{d z^{2}}=\frac{\varepsilon^{2}}{R^{3}}+\frac{2 I}{\mathrm{RI}_{A}(\beta \gamma)^{3}}
$$

where $R$ is beam radius (edge), $\varepsilon$ is the unnormalized edge emittance, and $I_{A}=17 \mathrm{kA}$. The cathode is in a magnetic field free region. We desire for the emittance to dominate space charge effects for the beamlets in the region following the pepperpot, i.e.

$$
\frac{\varepsilon_{n}^{2}}{R_{b}^{2}} \gg \frac{2 I}{\beta \gamma I_{A}} \text {, or } \varepsilon_{n}^{2} \gg \frac{I_{b} h^{2}}{2 \beta \gamma I_{A}} \text {, where } I=\left(\frac{h}{2 R_{b}}\right)^{2} I_{b} \text {. }
$$

$R_{b}$ and $I_{b}$ are the beam radius and current at the front of the pepperpot, and $h$ is the aperture. The size of the aperture is the only variable for adjusting the relative contribution of emittance to space charge. For the designed RTA injector beam parameters, $1.2 \mathrm{kA}, 1 \mathrm{MeV}$ and $100 \pi-\mathrm{mm}-\mathrm{mr}$, and using a $250-\mu \mathrm{m}$ aperture, the emittance term is approximately an order of magnitude larger than the space charge. Our aperture plate will consist of a rectangular pattern of 121 (11x11) $250-\mu \mathrm{m}$ apertures with $7 \mathrm{~mm}$ spacing on a $500-\mu \mathrm{m}$ thick tungsten plate. The tungsten plate represents about two range thickness for $1-\mathrm{MeV}$ electrons. A second 1-mm thick tungsten plate with nominal $750-\mu \mathrm{m}$ apertures will be placed in front of the thinner plate to improve thermal conduction. At four locations, apertures will not be drilled in the thicker plate to assist in determining background and orientation. The beamlets will strike a quartz foil located $80 \mathrm{~cm}$ (adjustable from 40 to $160 \mathrm{~cm}$ ) after the aperture plate. The Cherenkov light generated at the foil will be imaged with a gated camera. 
Small effects such as the aperture plate thickness (vignetting), fringe fields from the upstream solenoids, and beam waist location will be accounted for in the data analysis. A more serious problem concerns the effect of the conductive aperturc plate on the beam. It has been demonstrated (8) that, while the local distribution in phase space can be determined, the global $\mathrm{x}-\mathrm{x}$ ' curve is dominated by the non-linear focusing of the aperture plate. E-Gun simulations performed for our beam parameters indicate that the beam emittance determined from the pepperpot data will be as much as six times the actual emittance in the absence of the aperture plate. This effect can be accounted for in the analysis. However, the accuracy in the final emittance value will suffer. Note that this is only an issue for space charge dominated beams where aperturing is required.

An alternative to the pepperpot diagnostic is to vary the focusing of the beam and look at changes in the radial profile of the beam. The most straight forward method will be to insert a nonconducting foil a short distance after a solenoid. The strength of the solenoid could then be increased causing the focus to move through the foil. Matching the observed radius variation with the applied solenoidal field to computer simulations will allow the emittance to be inferred. Once again, the issue will be the minimum beam spot size the foil can tolerate. By operating in an over focus regime, i.e. the beam waist always remains in front of the foil, it may be possible to limit the minimum beam radius at the foil, but still generate significant variations in radius. We are also studying the effect of using a quadrupole magnet to focus in a single plane. This will reduce the issue of current density, but limit the emittance measurement to a single plane.

\section{RTA CONTROL SYSTEM}

Except for the fast beam diagnostics described above, the accelerator status is monitored through several Allen-Bradley SLC-500 logic controllers. The human interface is provided by National Instrument's BridgeView software running on a PC platform. Much of the pulsed power hardware, and accelerator support electronics is located adjacent to the gun. Initial pulsed power trials (without beam) have shown that the electromagnetic noise in the accelerator area associated with pulsing the gun does not disturb the control system. In tests on ETAII (with beam) the x-ray induced noise from spilt beam did not disturbed the control electronics. Additional testing will be needed to access the viability of the layout. The equipment has been placed near the gun to reduced the cost of the facility.

\section{ACCELERATOR SECTION}

Design of the full-scale RK-TBA system has an accelerator section after the gun that raises the beam energy from $1 \mathrm{MeV}$ to $2.5 \mathrm{MeV}$ before starting to bunch the beam at $11.4 \mathrm{GHz}$. In the accelerator section for a full scale RK-TBA we expect to use a pulse power system similar to that demonstrated in the gun system. The accelerator cells will be segmented to reduce the required drive. However, to reduce the cost of 
RTA we are planning on using a $120 \mathrm{kV}$ MARX spark-gap pulsed power system to drive 16 unsegmented accelerator cells in this section for RTA. This will still allow us to operate at a few $\mathrm{Hz}$ rate. The design is not acceptable for the full-scale machine because of the high gap erosion rates. The spark-gap system will provide a faster risetime than the thyratron system. The volt-second rating of the accelerator cores (operated at $120 \mathrm{kV}$ ) will limit the flat top of the beam pulse in the RTA. Installation of additional cells (future upgrade), and operating all the cells at a lower voltage, will increase the useful duration of the current pulse. We also plan to test a High-Gradient Insulator (9) in the first of these accelerator cells. If successful we would like to construct the 16 cells for RTA accelerator section using High-Gradient Insulators.

\section{ACKNOWLEDGMENTS}

The work was performed under the auspices of the U.S. Department of Energy by LLNL under contract W-7405-ENG-48, and by LBNL under contract AC0376SF00098. We thank Andy Sessler and Swapan Chattopadhyay for their support and guidance and thank Wayne Greenway, William Strelo, and Bob Candelario for their excellent technical support.

\section{REFERENCES}

1. Sessler, A.M. and Yu, S.S., "Relativistic Klystron Two-Beam Accelerator," Phys. Rev. Lett. 54, 889 (1987).

2. Westenskow, G.A., and Houck, T.L., "Relativistic Klystron Two-Beam Accelerator," IEEE Trans. on Plasma Sci., 22, 750 (1994).

3. Houck, T.L., and Westenskow, G.A., "Prototype Microwave Source for a Relativistic Klystron TwoBeam Accelerator" IEEE Trans. on Plasma Sci., 24, 938 (1996).

4. Registered name of Corning Glass Works.

5. Smith, C.H., and Barberi, L., "Dynamic Magnetization of Metallic Glasses," in Proc. of the 5th. IEEE Int'l Pulsed Power Conf., 1985.

6. Westenskow, G.A., et al., "Relativistic Klystron Two-Beam Accelerator Studies at the RTA Test Facility", Proc. of the 1997 Particle Accelerator Conference, Vancouver, Canada, 1997.

7. Houck, T.L., et al., "Diagnostics for a 1.2-kA, 1-MV Electron Induction Injector," Proc. of the $8^{\text {th }}$ Beam Instrumentation Workshop, SLAC, 1998.

8. Hughes, T.P., Carlson, R.L., and Moir, D.C., J. Appl. Phys. 68, 2562-2571 (1990).

9. Houck, T.L., et al., "Stacked Insulator Induction Accelerator Gaps", Proc. of the 1997 Particle Accelerator Conference, Vancouver, Canada, 1997. 